\title{
Construction of Stable Mouse Artificial Chromosome from Native Mouse Chromosome 10 for Generation of Transchromosomic Mice
}

Satoshi Abe

Tottori University

Kazuhisa Honma

Trans Chromosomics, Inc

Akane Okada

Tottori University

Kanako Kazuki

Tottori University

Hiroshi Tanaka

Trans Chromosomics, Inc

Takeshi Endo

Trans Chromosomics, Inc

Kayoko Morimoto

Trans Chromosomics, Inc

Takashi Moriwaki

Tottori University

Shusei Hamamichi

Tottori University

Yuji Nakayama

Tottori University

Teruhiko Suzuki

Tokyo Metropolitan Institute of Medical Science

Shoko Takehara

Trans Chromosomics, Inc

Mitsuo Oshimura

Tottori University

Yasuhiro Kazuki ( $\boldsymbol{\nabla}$ kazuki@tottori-u.ac.jp )

Tottori University 
Keywords: mouse artificial chromosome (MAC), microcell-mediated chromosome transfer (MMCT), chromosome engineering, transchromosomic (Tc) mouse, humanized model mouse

Posted Date: July 9th, 2021

DOI: https://doi.org/10.21203/rs.3.rs-675300/v1

License: (c) (1) This work is licensed under a Creative Commons Attribution 4.0 International License. Read Full License 


\section{Abstract}

Mammalian artificial chromosomes derived from native chromosomes have been applied to biomedical research and development by generating cell sources and transchromosomic (Tc) animals. Human artificial chromosome (HAC) is a precedent chromosomal vector which achieved generation of valuable humanized animal models for fully human antibody production and human pharmacokinetics. While humanized Tc animals created by HAC vector have attained significant contributions, there was a potential issue to be addressed regarding stability in mouse tissues, especially highly proliferating hematopoietic cells. Mouse artificial chromosome (MAC) vectors derived from native mouse chromosome 11 demonstrated improved stability, and they were utilized for humanized Tc mouse production as a standard vector. In mouse, however, stability of MAC vector derived from native mouse chromosome other than mouse chromosome 11 remains to be evaluated. To clarify the potential of mouse centromeres in the additional chromosomes, we constructed a new MAC vector from native mouse chromosome 10 to evaluate the stability in Tc mice. The new MAC vector was transmitted through germline and stably maintained in the mouse tissues without any apparent abnormalities. Through this study, the potential of additional mouse centromere was demonstrated for Tc mouse production, and new MAC is expected to be used for various applications.

\section{Introduction}

Human artificial chromosome (HAC) vectors derived from native human chromosomes, independently and stably maintained without disrupting host chromosomes, have capacities to carry desired copy number of genes via recombination sites, and transfer a large quantity of Mb-sized genes ${ }^{1}$. Taking these advantages over conventional vectors, transchromosomic (Tc) mice into which arbitrary genes were introduced via the HAC vectors, have been generated ${ }^{2,3}$. However, in an attempt to create a mouse model, the HAC vectors have demonstrated a variable retention rate in tissues of mouse individuals, and that stability is particularly low in hematopoietic cells with high proliferation ${ }^{4}$. To address this issue, we established a chromosome transfer- and recombinase-mediated genomic transfer (CT-RMGT) method that maintains an arbitrary human chromosomal region at the chromosome end of the mouse host via chromosome engineering technologies ${ }^{5}$. As a result, although stability was ensured, the method was labor-intensive and time-consuming, which was a barrier to constantly generate the mouse models.

Under these circumstances, a strategy to design a stable mouse artificial chromosome (MAC) vector was conceived through construction of a MAC vector from native mouse chromosome 11 based on the hypothesis that the centromere from own species contributes to high stability (hereinafter referred to as $11 \mathrm{MAC})^{4}$. As in the construction of HAC vectors, the MAC vectors have a structure in which endogenous gene region was deleted by telomere truncation, and the recombination acceptor site capable of gene loading and translocation cloning was inserted. The MAC vectors have the same advantages as the HAC vectors, and additionally have been shown to be uniformly and stably maintained in the mouse tissues including hematopoietic cells ${ }^{4}$. Several Tc mouse models for prediction of human pharmacokinetics and 
aneuploidy syndrome have been generated by using the $11 \mathrm{MAC}$ vectors ${ }^{6-9}$. Furthermore, the $11 \mathrm{MAC}$ was found to be stable in rat tissues by generating Tc rat models for human pharmacokinetics ${ }^{8}$.

Importantly, the 11MAC vector is stably maintained in the tissues of Tc mouse as the 41 st chromosome. In the Tc mice, two pairs of mouse chromosome 11 with endogenous genes and additional mouse chromosome 11 centromere with no genes are held. In particular, it has been revealed that the 11MAC vector itself does not affect development and homeostasis of the mouse ${ }^{3}$. However, it remains unclear whether MAC vector derived from native mouse chromosome other than mouse chromosome 11 are similarly and stably maintained in the mouse tissues as the 41 st chromosome. Further examples are needed to clarify the original hypothesis.

To investigate this, a new MAC with different centromere from other chromosomes excluding native mouse chromosome 11 was required. In addition, construction of MAC vector needed the native mouse chromosome tagged with drug selection marker to transfer the chromosome to other cells for efficient modification with conventional chromosome engineering techniques. In this study, to elucidate the above, we constructed a new MAC vector from native mouse chromosome 10 (10MAC), and produced Tc mice carrying the 10MAC. The 10MAC was constructed through the whole cell fusion and chromosome transfer with microcell-mediated chromosome transfer (MMCT) to the cells for chromosome modification. Constructed $10 \mathrm{MAC} 1$ and other divergent vectors were transferred to $\mathrm{CHO}$ cells for testing the gene loading capacity as gene delivery vectors. The 10MAC1 was further transferred to mouse embryonic stem cells to produce the Tc mice. Retention and structure of mouse chromosome 10 and $10 \mathrm{MAC}$ vectors in the host cells and mice were visibly analyzed by fluorescence in situ hybridization. We also confirmed that the 10MAC1 can be stably retained in the mouse tissues with GFP monitoring system and does not affect the development and homeostasis of the mouse. Taken together, we here demonstrated the generation of a functional MAC vector underived from mouse chromosome 11, and verified the capability of 10 MAC vectors for generation of Tc mice.

\section{Results}

\section{Generation of a $10 M A C$}

We performed the following experiments to generate MAC from native mouse chromosome 10 by chromosome engineering (Fig. 1). Mouse A9 cells are good donors for MMCT owing to the property of high micronuclei formation induced by colcemid treatment. Therefore, mouse embryonic fibroblasts, in which endogenous mouse chromosome 10 (mChr.10) was tagged with NeoR gene (mChr.10-NeoR), were fused with mouse A9 cells carrying Bsd gene. We obtained mouse A9-like whole cell hybrids resistant to both $\mathrm{G} 418$ and Blasticidin S. Then, the mChr.10-NeoR was transferred from the hybrid cells to homologous recombination-proficient chicken DT40 cells via MMCT. DT40 cells are useful host cells for efficient chromosome modification. FISH analysis confirmed that the mChr.10-NeoR was independently and stably maintained in DT40 hybrid cells (Fig. 2A). 
To truncate endogenous gene-coding region of $\mathrm{mChr} .10$, pericentromeric region was targeted by artificial telomere seeding technique (Fig. 2B). The targeting vector carrying the puromycin resistant gene was introduced into DT40 mChr.10-NeoR by electroporation. As a result of screening drug-resistant clones by PCR and FISH analyses, deletion of a region distal to the target sequence of $\mathrm{mChr} 10$ (hereafter referred as 10MAC) was confirmed in 4 out of 266 clones (DT40 10MAC) (Fig. 2C). In the obtained DT40 10MAC clones, deletion of the endogenous gene-coding region and NeoR gene resulted in an empty artificial chromosome composed of centromere and telomeres.

\section{Construction of MAC capable of gene loading by recombination system}

Consistent with the past artificial vectors we designed, we attempted to construct an artificial chromosome vector capable of carrying desired genes via Cre/loxP recombination system. First, a 10MAC vector carrying CAG-EGFP flanked by insulators, NeoR gene and loxP-3'HPRT was constructed (Fig. 2D). After electroporation of targeting construct to DT40 10MAC, drug selection was performed with G418, and 24 clones, positive for EGFP fluorescence and drug resistance, were randomly selected for further screening (Fig. 2E). Eight out of 24 clones were PCR positive, and as demonstrated by FISH analysis, in all 8 clones, a signal indicating the presence of the loaded unit was confirmed on the MAC maintained independently of the host chromosome (DT40 10MAC1) (Fig. 2F).

Next, 10MAC vector carrying NeoR gene and 5'HPRT-loxP was constructed (Fig. 3A). A targeting construct was introduced into DT40 10MAC by electroporation, and 23 clones were randomly selected from the obtained drug resistant clones. Twenty one out of 23 clones were PCR positive. Five clones were selected from PCR positive clones and FISH analysis was performed. We confirmed the presence of the platform inserted on the 10MAC that was maintained independently in all clones (DT40 10MAC2) (Fig. 3B).

Furthermore, the 10MAC which contains CAG-EGFP flanked by insulators, NeoR gene and 5'HPRT-loxP was constructed (Fig. 3C). A targeting construct was introduced into DT40 10MAC, and 24 EGFP fluorescence positive and drug resistant clones were randomly selected (Fig. 3D). PCR analysis was performed, and 23 clones were confirmed to be PCR positive. Among them, 5 clones were selected and analyzed by FISH. FISH analysis revealed that the expected targeting occurred on the 10MAC for all clones (DT40 10MAC3) (Fig. 3E). As a result, three types of 10MAC vectors with different platforms were constructed (Supplementary Fig. S1).

\section{Transfer of 10MAC1 to CHO hprt-/- cell line and verification of site-specific recombination}

The loxP-3'HPRT unit on the 10MAC1 is utilized for the reconstitution of HPRT gene and enables HAT selection when the desired gene with 5'-HPRT-loxP unit is loaded via Cre/loxP system. Therefore, 10MAC1 was transferred from DT40 to $\mathrm{CHO}$ hprt-/-, which is a Hprt gene-deficient cell line, by MMCT. Six clones of EGFP fluorescence positive and $\mathrm{G} 418$ resistant $\mathrm{CHO}$ hybrids were obtained (Fig. 4A). Although all clones were positive for PCR analysis, FISH analysis confirmed that one clone retained one copy of 10MAC1 independent of the host chromosome (CHO 10MAC1) (Fig. 4B). To evaluate whether the gene loading system functions on the 10MAC1, a plasmid carrying the 5'HPRT-IoxP unit and Cre expression vector were 
co-transfected into $\mathrm{CHO} 10 \mathrm{MAC1}$. PCR analysis of 24 randomly selected drug resistant clones revealed that all of them were positive for recombination junction PCR, which indicated that the system worked (Supplementary Table S2). Therefore, it was shown that the constructed 10MAC1 can be monitored by EGFP and gene loading can be performed efficiently.

\section{Transfer of 10MAC2 and 10MAC3 to CHO hprt-/- cells and verification of gene loading}

Following the strategy used to generate $10 \mathrm{MAC1}, 10 \mathrm{MAC} 2$ and $10 \mathrm{MAC} 3$ were transferred from DT40 cells to $\mathrm{CHO}$ hprt-/- cells by MMCT. Regarding the $10 \mathrm{MAC} 2,11$ out of 12 drug-resistant $\mathrm{CHO}$ hybrids were PCR positive. FISH analysis was performed on 6 randomly selected clones, and it was confirmed that 3 clones maintained one copy of the 10MAC2 independent from the host chromosome (Fig. 4C). For the 10MAC3, 9 out of 10 clones of EGFP fluorescence positive and drug resistant $\mathrm{CHO}$ hybrids were PCR positive (Fig. 4D). FISH analysis revealed that 2 out of 6 clones were desired clones carrying a single copy of the $10 \mathrm{MAC3}$ independent of the host (Fig. 4E).

For both 10MAC2 and 10MAC3, we verified whether gene-loaded clone could be obtained by HPRT reconstruction using the Cre/loxP system. A vector carrying loxP-3'HPRT and EGFP expression units and a vector containing loxP-3'HPRT and tdTomato expression units were transfected into $\mathrm{CHO} 10 \mathrm{MAC2}$ and CHO 10MAC3, respectively, with the Cre expression vector (Fig. 5A, 5D and Supplementary Table S2). We obtained HAT-resistant clones positive for EGFP and tdTomato fluorescence (Fig. 5B and 5E).

Furthermore, FISH analysis was performed to confirm the existence of on-board gene units on respective $10 \mathrm{MAC}$ vectors, excluding the possibility of random integration to host chromosome (Fig. $5 \mathrm{C}$ and $5 \mathrm{~F}$ ). Since signal of the loaded genes was detected on respective 10MACs, these results suggested that the gene-loading system worked as expected. Taken together, both 10MAC2 and 10MAC3 are capable of gene-loading using the Cre/loxP system.

\section{Transfer of the 10MAC1 vector to mouse embryonic stem cells and Tc mice generation}

To evaluate the stability of the 10MAC1 derived from native mouse chromosome 10 in mice, we attempted to generate Tc mouse carrying the 10MAC1. Chromosome transfer was performed by standard PEG-MMCT and retro-MMCT from CHO cells to mouse ES cells $(39, \mathrm{XO})$ and drug-resistant and GFPpositive clones were selected for further analyses (Fig. 6A). Retro-MMCT is a highly efficient chromosome transfer method reported recently, which can reduce donor scale. FISH analysis with the digoxigeninlabeled mouse minor satellite and biotin-labeled platform construct ( $\mathrm{pVGNLH1)}$ probes showed that a single copy of $10 \mathrm{MAC} 1$ was independently maintained in mES hybrids (Fig. 6B). Among them, we selected clones with desired karyotype $(40, \mathrm{XO},+10 \mathrm{MAC} 1)$ (TT2F 10MAC1). Then, we produced female chimeric mice with high coat-color chimerism by injecting TT2F 10MAC1 into eight-cell-stage embryo. These chimeric mice were crossed with wild-type mice to verify whether the $10 \mathrm{MAC} 1$ derived from native mouse chromosome 10 is transmitted through germline. Among the offsprings, GFP-positive mice with the 10MAC1 retention were obtained without any apparent abnormality (Fig. 6C). To confirm the independent maintenance of $10 \mathrm{MAC} 1$ from host chromosome, the metaphase spreads of lymphocytes in blood collected from Tc mice were analyzed. The 10MAC1 was single copy and independently 
maintained in host cells without integration (Fig. 6D). These results suggested that the 10MAC1 can be transmitted through germline and independently maintained without affecting the development of Tc mouse.

\section{Retention rate of the 10MAC1 vector in mouse}

To investigate whether 10MAC1 derived from native mouse chromosome 10 is stable in the mouse tissues in the identical manner as previously constructed 11MAC derived from native mouse chromosome 11, we analyzed Tc mice carrying 10MAC1 vector. The EGFP on the MAC1 was expressed in all tissues examined, which is comparable to 11MAC Tc mice (Fig. 6E).

Given the high rate of cellular turnover, hematopoietic cells from blood, bone marrow and spleen are optimal target cells to evaluate the stability of mammalian artificial chromosomes (ACs) in mouse. In previous report, HAC vector was relatively stable in various tissues except hematopoietic cells in Tc mice. In contrast, $11 \mathrm{MAC}$ derived from native mouse chromosome 11 is highly stable in all tissues examined including hematopoietic cells in Tc mice ${ }^{4}$. The degree of stability is thought to be more pronounced in the hematopoietic cells with rapid turnover. According to the data from several Tc mouse models generated by HAC and MAC vectors, the retention rate of mammalian ACs in the hematopoietic cells reflects those of mammalian ACs in the whole body.

Considering the reports mentioned above, in this study, we evaluated the stability of 10MAC1 in Tc mice by monitoring GFP-positive cells from blood, bone marrow and spleen by FCM analysis. The stability of $10 \mathrm{MAC} 1$ can be evaluated by monitoring GFP-positive cells because the $10 \mathrm{MAC} 1$ contains the GFP expression unit driven by constitutive active CAG promoter in common with the MAC as reported previously. The retention rate of the 10MAC1 was high and uniform in B cells, CD4 and CD8 T cells from peripheral blood, bone marrow and spleen (89-94\%) (Fig. 6F). The rates of GFP-positive cells with the

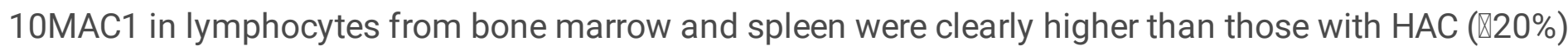
and comparable to those with MAC ( $\triangle 90 \%)$. The transmission efficiency and stability of $10 \mathrm{MAC} 1$ in lymphocytes was evaluated through generations. The transmission rate from male and female was $11.7 \%$ and $32.1-69.2 \%$, respectively. Regarding the stability, although some Tc mice showed lower retention rate, main population maintained high stability through generations (Supplementary Fig. S2). These results suggested that the $10 \mathrm{MAC} 1$ derived from native mouse chromosome 10 is highly stable in mice.

\section{Discussion}

In this study, we constructed a new MAC vector from native mouse chromosome 10. The 10MAC1 was transmitted through germline in mice and there was no apparent abnormality in Tc mice carrying $10 M A C 1$ through generations. The assessment of stability by FCM analysis of lymphocyte subsets from each tissue clarified the high stability of $10 \mathrm{MAC} 1$ even in the hematopoietic cells with high proliferation. From the previous reports, because of the correlation between high proliferation and increased loss of 
mammalian ACs, the stability of artificial chromosomes in lymphocyte subset is well established determinant factor reflecting their stability among various tissues in the host individuals ${ }^{4}$. Therefore, $10 \mathrm{MAC} 1$ is expected to be available for generating Tc mouse models. The study also indicated that the MAC derived from native mouse chromosome other than mouse chromosome 11 is also highly stable in mice.

Further study is necessary to evaluate the different degrees of stability levels among native mouse centromeres by generating various MACs from other native chromosomes. To this end, multiple technological approaches through the combinations of the recent advancements in genome editing technology and chromosome transfer are expected to promote the research regarding the stability of centromeres in own species. In this analysis, as a starting point to clarify the potential of native mouse centromere for Tc mice production, the MAC vectors constructed herein can be utilized for various purposes including: 1) efficient gene loading system considering compatible constructs prepared previously, 2) cell resource production by transfer of new MACs with functional gene units, and 3) investigation on the compatibility of centromere function beyond species.

The 10MAC vectors described herein offer the following notable features with distinct aims. The 10MAC1 with loxP-3'HPRT is aimed at translocation cloning of desired chromosome fragments with 5'HPRT-loxP such as human native chromosome region to generate a humanized mouse model with gene cluster of Mb-sized gene ${ }^{10}$. The $10 \mathrm{MAC} 2$ is for loading general single vector or previously established simultaneous or sequential integration of multiple gene loading vectors (SIM system) which enable us to load multiple vectors sequentially or three vectors simultaneously each round ${ }^{10,11}$. The other gene loading system such as multi-integrase (MI) system also can be applied to 10MAC2 by inserting MI platform with four integrase and one recombinase acceptor sites ${ }^{12-14}$. The 10MAC3 is a chromosomal vector in which an EGFP monitor system is added to $10 \mathrm{MAC2}$.

The 10MAC2 and 10MAC3 are expected to be utilized for not only generating mouse models but also establishing useful cell lines for material production and functional analysis comparable to those described by using $11 \mathrm{MAC}^{4}$. Several in vitro models have already been established; i.e., hepatoblastoma cell line, HepG2 carrying the 11MAC with the drug-metabolizing cytochrome P450 (CYP) enzymes for assessing drug metabolism and hepatotoxicity ${ }^{15-17}$, human colon adenocarcinoma cell line, Caco2 carrying the MAC with CYPs for prediction of the absorption and metabolism in the human intestine ${ }^{18}$, luminescent HepG2 with the MAC carrying luciferase reporter units for cytotoxicity assay ${ }^{17}$, immortalized mouse S3 cells carrying the 11MAC with Kidney injury molecule-1 (Kim-1) and Hprt reporter units for assessing nephrotoxicity ${ }^{19}$, and human mesenchymal stem cells (MSCs) carrying the MAC with osteocalcin (OC) reporter for monitoring osteogenic differentiation ${ }^{20}$. Additionally, recent work reported a double 11MAC-retaining cell based model wherein HepG2 cells with multiple CYPs and POR genes on one $11 \mathrm{MAC}$ and luminescence expression unit on another were generated for easy real-time monitoring system of hepatotoxicity by molecules ${ }^{21}$. Evidence of their applications with multiple copies also suggested the flexibility of MAC vector system. 
The stability of 10MAC vectors in cells and tissues of other species remains to be evaluated. As with $11 \mathrm{MAC}$ vectors, $10 \mathrm{MAC}$ vectors are presumably stable in human cell lines such as fibrosarcoma HT1080

and hepatoblastoma HepG $2^{16,22}$. In addition, high stability of the $11 \mathrm{MAC}$ vectors in the humanized Tc rat models for pharmacokinetics implies the potential of 10MAC vectors for generation of Tc rat models ${ }^{8}$. Establishment of 10MAC-containing cell lines derived from several species may promote investigation on the compatibility between mouse centromere and centromere-associated proteins of host species to search the key factors for chromosome stability. Interestingly, on stability of human chromosomes in the mouse cell line, while human chromosomes 2 and 11 were unstable in the mouse ES cells, HAC derived from human chromosome 14 (SC20) was stable when compared with those chromosomes leading to the concept of the link between the stability and composition of centromeres even in interspecies comparability ${ }^{23}$. Since the study cannot exclude the effect of endogenous genes of intact or partially fragmented chromosomes on stability, constructions of mammalian ACs without endogenous genes may also promote such analyses.

In conclusion, although universal mammalian AC vector, which is highly stable among the species, is still desired and certain chromosomal vectors such as 11MAC is known to be available for mouse and rat, this study further provided the evidence supporting the original conception that construction of artificial chromosomes from own species is also reasonable strategy for generation of Tc animals from desired species. Collectively, the 10MAC vectors constructed in this study is expected to contribute to generation of Tc mouse models and valuable cell resources for biomedical research and development.

\section{Materials And Methods}

\section{Ethics statement}

This study was approved by the Institutional Animal Care and Use Committee of Tottori University and the Recombinant DNA Experiment safety Committee of Tottori University (for performing recombinant DNA experiments). All experiments were carried out in compliance with the ARRIVE guidelines. All methods were carried out in accordance with relevant guidelines and regulations.

\section{Cell Culture}

The mouse embryonic fibroblasts, mouse A9 cells, and whole cell hybrids were grown in Dulbecco's modified Eagle's medium (DMEM; Wako, Tokyo, Japan) supplemented with 10\% fetal bovine serum (FBS; Sigma-Aldrich, St. Louis, MO, USA). The DT40 hybrid cells were maintained in Roswell Park Memorial Institute (RPMI) medium 1640 (Wako, Tokyo, Japan) containing 10\% FBS, 1\% chicken serum (Gibco, ThermoFischer, Waltham, MA, USA), $50 \mu \mathrm{mol} / \mathrm{L} 2$-mercaptoethanol, and the appropriate antibiotics. The DT40 hybrids containing a single copy of mouse chromosome 10 were produced by MMCT from mouse A9 hybrid cells containing NeoR-tagged mouse chromosome 10 and maintained with $1500 \mu \mathrm{g} / \mathrm{mL}$ G418 (Promega, Madison, WI, USA). The hypoxanthine phosphoribosyltransferase (Hprt)-deficient $\mathrm{CHO}$ (JCRB0218) hybrids containing a 10MAC1, 10MAC2 or 10 MAC3 were maintained in Ham's F-12 nutrient 
mixture (Wako, Tokyo, Japan) containing 10\% FBS and $800 \mu \mathrm{g} / \mathrm{mL}$ G418. The parental mouse ES cell line, TT2F, and the microcell hybrid clone, TT2F (10MAC1), were maintained on mitomycin C (SigmaAldrich, St. Louis, MO, USA)-treated neomycin-resistant MEFs (Oriental Yeast, Tokyo, Japan) as feeder layers in DMEM with 18\% FBS, 1 mM sodium pyruvate (Invitrogen, Carlsbad, CA, USA), $0.1 \mathrm{mM}$ nonessential amino acids (Invitrogen, Carlsbad, CA, USA), $0.1 \mathrm{mM}$ 2-mercaptoehtanol (Sigma-Aldrich, St. Louis, MO, USA), 2 mM L-glutamine (Invitrogen, Carlsbad, CA, USA), and 1,000 units/mL leukemia inhibitory factor (Funakoshi, Tokyo, Japan).

\section{Construction of Targeting vectors}

The homologous regions on mouse chromosome 10 are described in Figure 1. For constructing a telomere truncation vector, pBS-TEL/puro_10MAC, annealed sense and antisense EcoRI/Ascl/EcoRI oligos were inserted into EcoRI site of pBS-TEL/Puro vector ${ }^{24}$. Then, a fragment (5.6 kb) of the mouse chromosome 10 region was amplified by PCR with Ascl_m10T F2/BamHI_m10T R3 primers, digested with Ascl/BamHI and sub-cloned into the Ascl/BamHI sites of the pBS-TEL/Puro vector (pBSTEL/puro_10MAC). The targeting vector, p10MAC1, for introducing EGFP/neo/loxP-3'HPRT was constructed as follows: Two $1.4 \mathrm{~kb}$ and $4.4 \mathrm{~kb}$ fragments for homologous arms corresponding to the mouse chromosome 10 pericentromeric region were amplified by PCR using the Kpnl_m10 LA F/Xhol_m10 LA R primers (2.0 kb) and Sall_m10 RA F/ Notl_m10 RA R primers (4.4 kb), digested with $\mathrm{Kpnl} / \mathrm{Xhol}$ and Sall/Notl, and subcloned into pKO Scrambler V913 backbone vector (Lexicon Genetics, Woodlands, TX, USA). Then, the EGFP/neo/3'HPRT-loxP cassette from pVGNLH1 ${ }^{4}$, digested with Sall/Ascl, was introduced into Xhol/Ascl sites of the vector (p10MAC1). The targeting vector, p10MAC2, for introducing neo/5'HPRT-loxP was constructed as follows: PGKneo and 5'HPRT-loxP fragments were introduced into EcoRI site and Ascl/Clal sites of pKO Scrambler V907 backbone vector (Lexicon Genetics, Woodlands, TX, USA). Then, a $4.4 \mathrm{~kb}$ right arm was amplified by PCR using Clal_m10 RA F/R primers and inserted into Clal site of the vector (pN5'HLR). A $2.0 \mathrm{~kb}$ left arm was amplified by Notl_m10 LA F/Sall_m10 LA R primers and inserted into Notl/Sall sites of pN5'HLR (p10MAC2). The targeting vector, p10MAC3, for introducing EGFP/neo/5'HPRT-loxP was constructed as follows: CAG-EGFP flanked by insulator HS4 was inserted to Notl/Sall sites of pN5'HLR (pN5'HLER). Then, left arm was amplified by Notl_m10 LA F/Sall_m10 LA R primers and a $1.9 \mathrm{~kb}$ Notl/PspOMI-digested fragment was introduced to Notl site of the pN5'HLER (p10MAC3). Primer information is available in Supplementary Table S1.

\section{Transfection of DT4O and $\mathrm{CHO}$ cells}

The DT40 (mChr.10-neo) hybrid cells were transfected by electroporation of $1 \times 10^{7}$ cells with each Notllinearized plasmid at $25 \mu \mathrm{F}$ and $550 \mathrm{~V}$ in a $4 \mathrm{~mm}$ cuvette using a Gene Pulser (Bio-Rad, Hercules, CA). The cells were resuspended in basic growth medium and aliquoted into 96-well flat-bottomed microtiter plates with serial dilution (Becton-Dickinson, Franklin Lakes, $\mathrm{NJ}$ ). Next day, the cells were resuspended in selective medium with $0.5 \mu \mathrm{g} / \mathrm{ml}$ puromycin or $1.5 \mathrm{mg} / \mathrm{ml} \mathrm{G} 418$. Approximately fourteen days later, drugresistant colonies were picked up and expanded for the following analyses. The $\mathrm{CHO}$ cells $\left(2 \times 10^{6} \mathrm{cells}\right)$ containing 10MAC1, 10MAC2 or 10MAC3 were transfected with pBS185 (CMV-Cre) and plasmids 
containing 5'HPRT-IoxP (X6.1), loxP-3'HPRT-I-CAG-EGFP-I (X3.1-I-EGFP-I) or loxP-3'HPRT-I-EF1a-tdTomato-I (X3.1-I-tdTomato-I) by using $20 \mu \mathrm{L}$ of Lipofectamine 2000 reagent (Invitrogen, Carlsbad, CA, USA) according to the manufacture's instruction. After $24 \mathrm{~h}$, cells were scaled up, and after $48 \mathrm{~h}$, cultured in the medium containing $1 \times$ HAT (Sigma-Aldrich, St. Louis, MO, USA) for selection. Fourteen days later, drugresistant colonies were picked up and expanded for further analyses as described below.

\section{MMCT}

The 10MAC1, 10MAC2 and 10MAC3 vectors were transferred from the DT40 cells into the CHO hprt-/cells using MMCT technology ${ }^{2}$. Briefly, microcells were prepared by centrifugation of DT40 cells attached to flasks (Nalge Nunc, Rochester, NY, USA) coated with poly-L-lysine followed by fusion with $1 \times 10^{6} \mathrm{CHO}$ cells using $42 \%$ polyethylene glycol 1000 (Wako, Tokyo, Japan). CHO hybrids containing each 10MAC vector were selected with the media containing $800 \mu \mathrm{g} / \mathrm{mL}$ G418 and selected for expansion. CHO cells containing 10MAC1 without and with ecotropic Env $\triangle \mathrm{R}$ expression were used as donor microcell hybrids in the PEG-MMCT and retro-MMCT as described previously ${ }^{25,26}$. mES XO ES9 hybrids containing $10 \mathrm{MAC} 1$ were selected with the media containing $150 \mu \mathrm{g} / \mathrm{mL}$ G418 and picked for expansion.

\section{Genomic PCR analysis}

Genomic DNA was extracted from cell lines using a genomic extraction kit (Gentra Systems, Minneapolis, MN, USA), and PCR was performed as follows. The screening primers for telomere truncation were $\mathrm{m} 10$ F6/Purol, Gm8155 F/R, lyd F/R and Plekhg1 F/R. The targeting with p10MAC1 was confirmed by using KpnI_m10 LA F/Xhol m10 LA R, m10 F1/EGFP-F and kj neo/m10 R2. The primers for detection of exact targeting with p10MAC2 were m10 F1/10MAC R1 and 10MAC F1/m10 R2. The targeted allele by p10MAC3 was analyzed by using m10 F1/EGFP-R and 10MAC F1/m10 R2. Contamination of DT40 cells after MMCT from DT40 cells to $\mathrm{CHO}$ cells were checked by using CENPH Fw/Rv. Contamination of $\mathrm{CHO}$ cells after MMCT from $\mathrm{CHO}$ cells to mES cells were checked by using Furin F/R. The recombination with Cre/loxP system on each 10MAC vector was detected by TRNS L1/R1. Primer sequences are described in Supplementary Table S1.

\section{Fluorescence in situ hybridization (FISH) analysis}

Preparation of metaphase chromosomes from exponentially growing cell culture was performed according to standard methods. FISH analyses were performed using the digoxigenin-labeled (Roche, Basel, Switzerland) mouse COT-1 DNA (Invitrogen, Carlsbad, CA, USA) or mouse minor satellite DNA, the biotin-labeled PGKpuro, pVGNLH1, X6.1, X3.1-I-EGFP-I, or X3.1-I-tdTomato-I plasmid DNA essentially as described previously ${ }^{26}$. Chromosomal DNA was counterstained with DAPI (Sigma-Aldrich, St. Louis, MO, US). Images were captured using an AxiolmagerZ2 fluorescence microscope (Carl Zeiss, Oberkochen, Germany).

\section{Generation of chimeric mice}


Chimeric mice were produced from TT2F hybrids containing 10MAC1. Chimera production was performed as described previously ${ }^{26}$. mES cells were injected into 8-cell stage embryos derived from ICR mice (CLEA, Tokyo, Japan) and then transferred into pseudopregnant ICR females. Almost 100\% coatcolor chimeric mice were mated with ICR males to obtain Tc mice.

\section{GFP image capture and FCM analysis of tissues and blood cells}

Samples were collected from euthanized Tc mice containing 10MAC1 and their 10MAC1-negative counterpart with isoflurane after perfusion. Bright and GFP images of each tissue were obtained by M205 FA fluorescence stereo microscope (Leica Microsystems, Wetzlar, Germany) with NIS-Elements software (Nikon, Tokyo, Japan). The dissociated cells were treated with ammonium chloride solution for hemolysis and stained with anti-mouse B220/CD45R (Biolegend, San Diego, CA, USA), CD3 (Biolegend, San Diego, CA, USA), CD4 (Biolegend, San Diego, CA, USA) and CD8a (BD Biosciences, San Jose, CA, USA) antibodies conjugated with BV650, BV650, PE and PE-CF594, respectively. All staining samples ( $1 \times 10^{6}$ cells) were incubated at $4{ }^{\circ} \mathrm{C}$ for $30 \mathrm{~min}$ in $100 \mu \mathrm{FCM}$ buffer (HBSS with 5\% FBS and $1 \mathrm{mM}$ EDTA) (Gibco, ThermoFischer, Waltham, MA, USA). DAPI was added to the final suspension to exclude dead cells. Analyses were conducted using a CytoFLEX S (Beckman Coulter, Brea, CA, USA). Percentage of GFPexpressing cells in lymphocytes through generations was analyzed by Gallios (Beckman Coulter, Brea, CA, USA).

\section{Declarations}

\section{Acknowledgments}

We thank Toko Kurosaki, Yukako Sumida, Tomoko Ashiba, Kei Yoshida, Eri Kaneda, Masami Morimura, Michika Fukino, Megumi Hirose and Fumihiko Adachi at Tottori University and Wakako Kotoku, Shinya Komoto, Rina Ohnishi, Akane Morita and Moeka Koshima at Trans Chromosomics Inc. for technical assistance. We also thank Dr. Hiroyuki Kugoh, Dr. Masaharu Hiratsuka, Dr. Hiroyuki Satofuka and Dr. Takahito Ohira at Tottori University for critical discussions. This research was partly performed at the Tottori Bio Frontier managed by Tottori prefecture. The work was supported in part by JST CREST Grant Number JPMJCR18S4, Japan (Y.K.), the Basis for Supporting Innovative Drug Discovery and Life Science Research (BINDS) of Japan Agency for Medical Research and Development (AMED) under grant number JP21am0101124 (YK), and the Science and Technology Platform Program for Advanced Biological Medicine from AMED under Grant Number JP21am0401002 (Y.K.).

\section{Author contributions}

S.A. participated in all aspects and wrote the manuscript. K.K. obtained DT40 hybrids with tagged mouse chromosome 10. T.E. constructed vectors. A.O. and H.T. performed chromosome modification. A.O., K.H. and T.S. cultured cells and performed MMCT. K.K. and K.H. performed FISH analyses. K.M. performed generation, maintenance, and analyses of Tc mice. T.M., K.H. and Y.N. performed FCM analyses. S.H. revised the manuscript. S.T., M.O. and Y.K. supervised this study. All authors reviewed the manuscript. 


\section{Competing Interests Statement}

M.O. is a CEO, employee and shareholder of Trans Chromosomics, Inc. S.A., K.H., H.T., T.E., K.M. and S.T. are employees of Trans Chromosomics, Inc., and the other authors declare no conflicts of interest.

\section{Data availability}

The datasets generated during and/or analyzed during the current study are available from the corresponding author on reasonable request.

\section{References}

1. Oshimura, M., Uno, N., Kazuki, Y., Katoh, M. \& Inoue, T. A pathway from chromosome transfer to engineering resulting in human and mouse artificial chromosomes for a variety of applications to bio-medical challenges. Chromosome Research vol. 23 111-133 (2015).

2. Kuroiwa, Y. et al. Manipulation of human minichromosomes to carry greater than megabase-sized chromosome inserts. Nature Biotechnology vol. 18 1086-1090 (2000).

3. Kazuki, Y. et al. Trans-chromosomic mice containing a human CYP3A cluster for prediction of xenobiotic metabolism in humans. Human Molecular Genetics vol. 22 578-592 (2013).

4. Takiguchi, M. et al. A novel and stable mouse artificial chromosome vector. ACS Synthetic Biology vol. 3 903-914 (2014).

5. Takehara, S. et al. A novel transchromosomic system: Stable maintenance of an engineered Mbsized human genomic fragment translocated to a mouse chromosome terminal region. Transgenic Research vol. 23 441-453 (2014).

6. Yamasaki, Y. et al. Characterization of P-glycoprotein humanized mice generated by chromosome engineering technology: Its utility for prediction of drug distribution to the brain in humans. Drug Metabolism and Disposition vol. 46 1756-1766 (2018).

7. Abe, S. et al. Modification of single-nucleotide polymorphism in a fully humanized CYP3A mouse by genome editing technology. Scientific Reports vol. 7 1-11 (2017).

8. Kazuki, Y. et al. Humanized UGT2 and CYP3A transchromosomic rats for improved prediction of human drug metabolism. Proceedings of the National Academy of Sciences of the United States of America vol. 116 3072-3081 (2019).

9. Kazuki, Y. et al. A non-mosaic transchromosomic mouse model of down syndrome carrying the long arm of human chromosome 21. eLife vol. 9 1-29 (2020).

10. Uno, N., Abe, S., Oshimura, M. \& Kazuki, Y. Combinations of chromosome transfer and genome editing for the development of cell/animal models of human disease and humanized animal models. Journal of Human Genetics vol. 63 145-156 (2018).

11. Suzuki, T., Kazuki, Y., Oshimura, M. \& Hara, T. A novel system for simultaneous or sequential integration of multiple gene-loading vectors into a defined site of a human artificial chromosome. 
PLOS ONE vol. 9 (2014).

12. Yamaguchi, S. et al. A method for producing transgenic cells using a multi-integrase system on a human artificial chromosome vector. PLOS ONE vol. 6 (2011).

13. Yoshimura, Y. et al. Mouse embryonic stem cells with a multi-integrase mouse artificial chromosome for transchromosomic mouse generation. Transgenic Res. 24, 717-727 (2015).

14. Honma, K. et al. Development of a multiple-gene-loading method by combining multi-integration system-equipped mouse artificial chromosome vector and CRISPR-Cas9. PLoS One 13, 1-19 (2018).

15. Takenaka, T. et al. Development of Caco-2 cells co-expressing CYP3A4 and NADPH-cytochrome P450 reductase using a human artificial chromosome for the prediction of intestinal extraction ratio of CYP3A4 substrates. Drug Metab. Pharmacokinet. 32, 61-68 (2017).

16. Satoh, D. et al. Establishment of a novel hepatocyte model that expresses four cytochrome P450 genes stably via mammalian-derived artificial chromosome for pharmacokinetics and toxicity studies. PLoS ONE vol. 12 1-18 (2017).

17. Satoh, D. et al. Human and mouse artificial chromosome technologies for studies of pharmacokinetics and toxicokinetics. Drug Metab. Pharmacokinet. 33, 17-30 (2018).

18. Ohta, Y. et al. Development of Caco-2 cells expressing four CYPs via a mammalian artificial chromosome. BMC Biotechnol. 20, 1-10 (2020).

19. Kokura, K. et al. A kidney injury molecule-1 (Kim-1) gene reporter in a mouse artificial chromosome: the responsiveness to cisplatin toxicity in immortalized mouse kidney S3 cells. J. Gene Med. 18, 273-281 (2016).

20. Narai, T. et al. Construction of a luciferase reporter system to monitor osteogenic differentiation of mesenchymal stem cells by using a mammalian artificial chromosome vector. Yonago Acta Med. 58, 23-29 (2015).

21. Iwado, S., Abe, S., Oshimura, M., Kazuki, Y. \& Nakajima, Y. Bioluminescence measurement of timedependent dynamic changes of cyp-mediated cytotoxicity in cyp-expressing luminescent hepg2 cells. Int. J. Mol. Sci. 22, 1-14 (2021).

22. Kazuki, K. et al. Highly stable maintenance of a mouse artificial chromosome in human cells and mice. Biochemical and Biophysical Research Communications vol. 442 44-50 (2013).

23. Shinohara, T. et al. Stability of transferred human chromosome fragments in cultured cells and in mice. Chromosom. Res. 8, 713-725 (2000).

24. Kuroiwa, Y. et al. Efficient modification of a human chromosome by telomere-directed truncation in high homologous recombination-proficient chicken DT40 cells. Nucleic Acids Research vol. 26 3447-3448 (1998).

25. Suzuki, T., Kazuki, Y., Oshimura, M. \& Hara, T. Highly efficient transfer of chromosomes to a broad range of target cells using Chinese hamster ovary cells expressing murine leukemia virus-derived envelope proteins. PLoS ONE vol. 11 1-11 (2016). 
26. Tomizuka, K. et al. Functional expression and germline transmission of a human chromosome fragment in chimaeric mice. Nature Genetics vol. 16 133-143 (1997).

\section{Figures}

(1) Cloning of a mouse chromosome and construction of the 10MAC

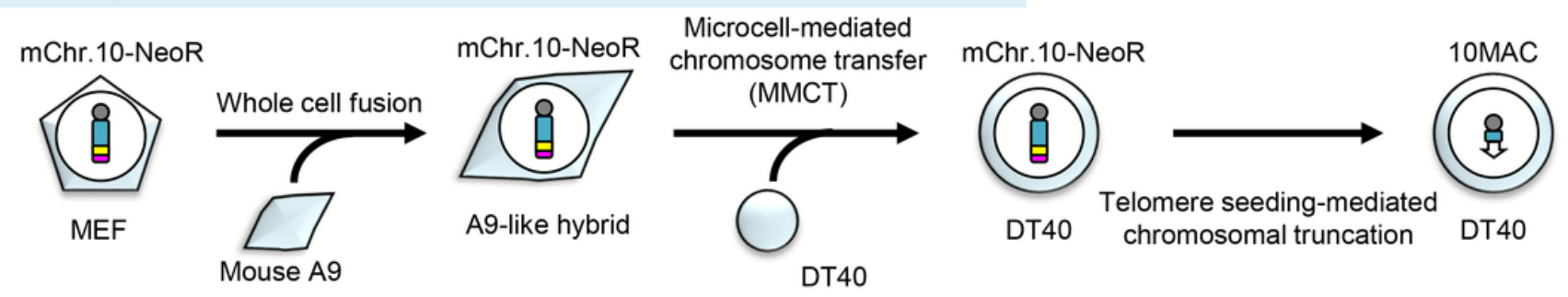

(2) Construction of the 10MAC1 and production of Tc mouse

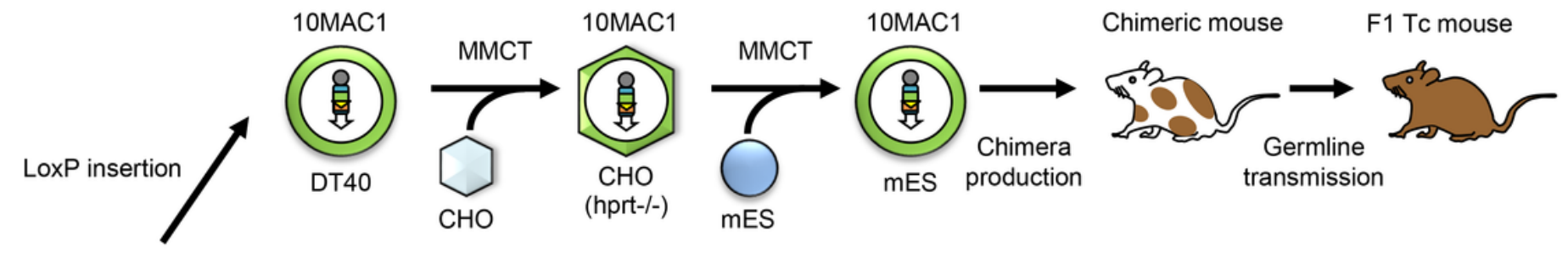

(3) Construction of the 10MAC2 and 10MAC3, and gene loading

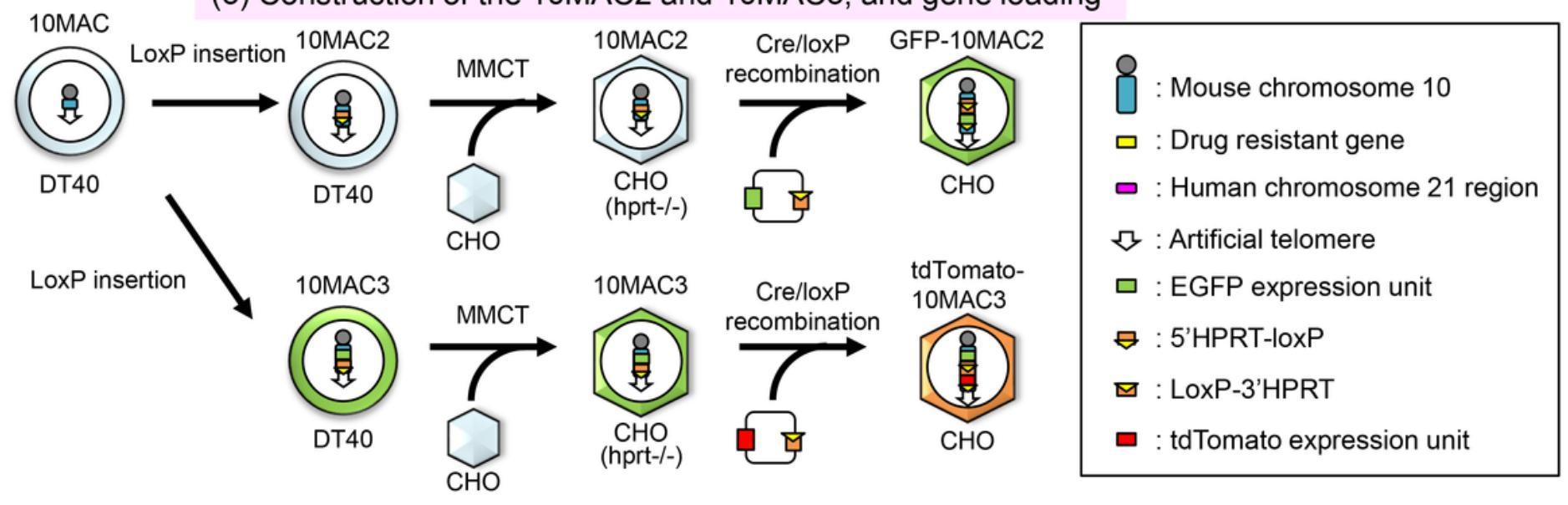

\section{Figure 1}

Schematic diagram outlining the construction of new MAC vectors (1) Cloning of a mouse chromosome and construction of the 10MAC vector. Mouse embryonic fibroblasts (MEFs) containing the NeoR-tagged mouse chromosome 10 (mChr.10-NeoR) were fused with mouse A9 cells. The mChr.10-NeoR was transferred from whole cell hybrids into DT40 cells by microcell-mediated chromosome transfer (MMCT), and the DT40 microcell hybrids containing the mChr.10-NeoR were designated DT40 mChr.10-NeoR. Chromosome manipulation was performed in the homologous recombination-proficient DT40 microcell hybrids. The distal q-arm was deleted from the mChr.10-NeoR by telomere seeding-mediated chromosomal truncation. This mini-chromosome was designated as 10MAC. (2) Construction of the 10MAC1 vector and production of Tc mice. The plasmid containing EGFP and loxP-3'HPRT genes were 
cloned into a specific site of the 10MAC vector in DT40 cells by homologous recombination. The MAC vector containing EGFP and loxP site was designated as $10 \mathrm{MAC1}$ vector. The EGFP can monitor the existence of $10 \mathrm{MAC} 1$ vector. The $10 \mathrm{MAC} 1$ was transferred into mouse embryonic stem (ES) cells via $\mathrm{CHO}$ cells for subsequent studies. To investigate the stability of the 10MAC1 vector, chimeric mice were produced from the ES cells containing the 10MAC1. The F1 mice were obtained by mating between chimeric and wild-type mice. (3) Construction of the 10MAC2 and 10MAC3 vectors and Cre/loxPmediated gene loading. The 5'HPRT-loxP plasmid was targeted to a proximal region of the q-arm of the $10 \mathrm{MAC}$ vector in DT40 cells (10MAC2). The 10MAC2 vector was transferred into $\mathrm{CHO}$ (hprt-/-) cells and the circular vector carrying EGFP and loxP-3'HPRT was loaded into the 10MAC2 vector. The plasmid carrying EGFP and 5'-HPRT loxP was targeted to the MAC vector in DT40 cells (10MAC3). The 10MAC3 vector was transferred into $\mathrm{CHO}$ (hprt-/-) cells and the circular vector containing loxP-3'HPRT and tdTomato was loaded into the 10MAC3 vector.

A

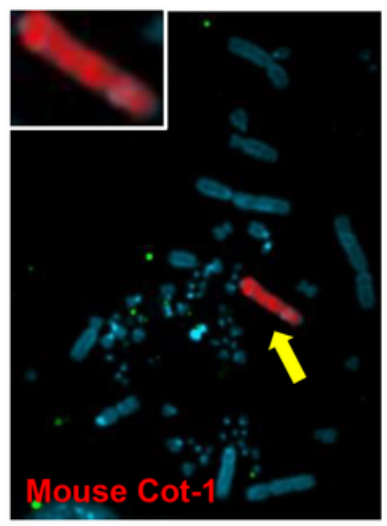

B

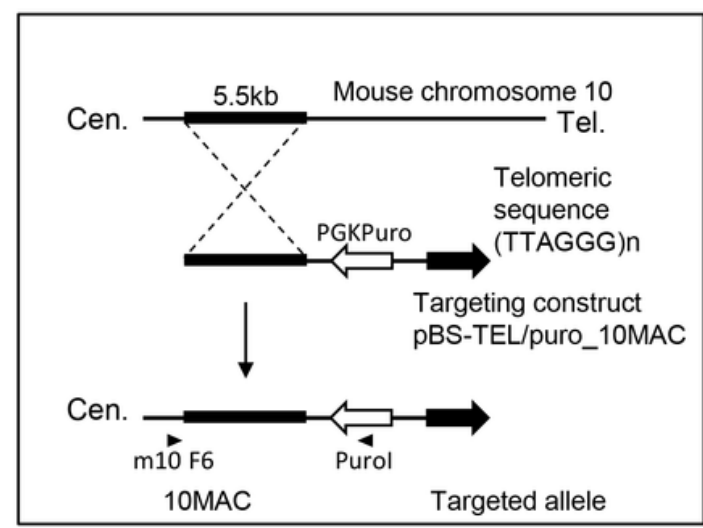

$E$

D

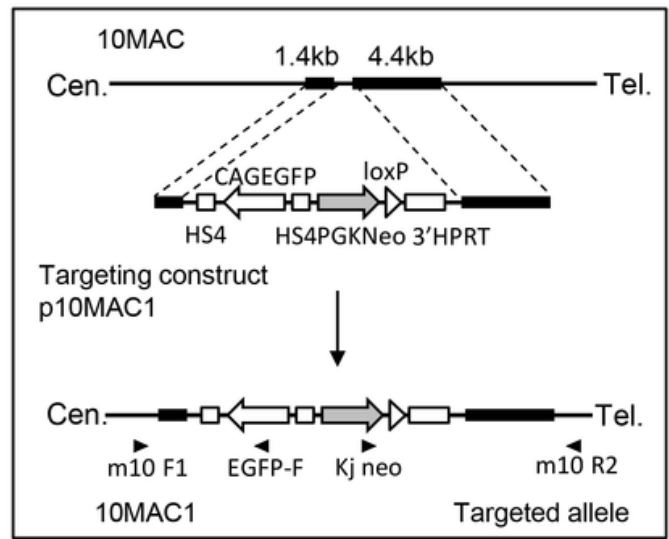

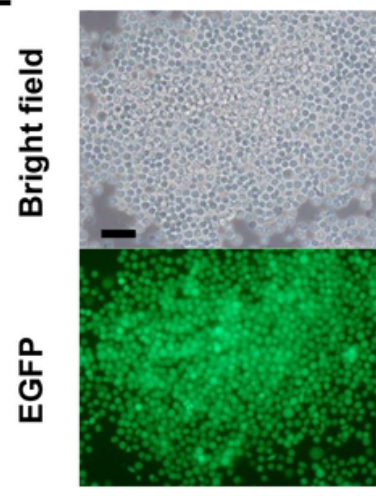

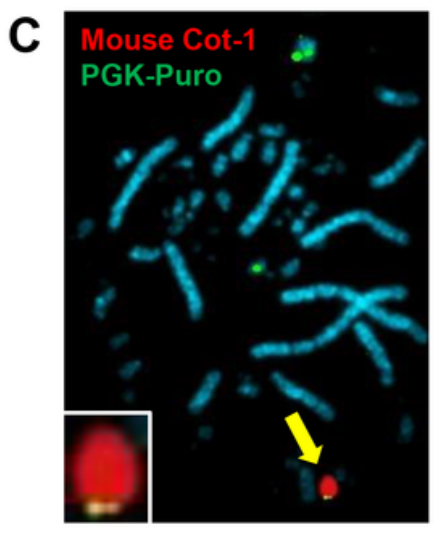

$\mathbf{F}$

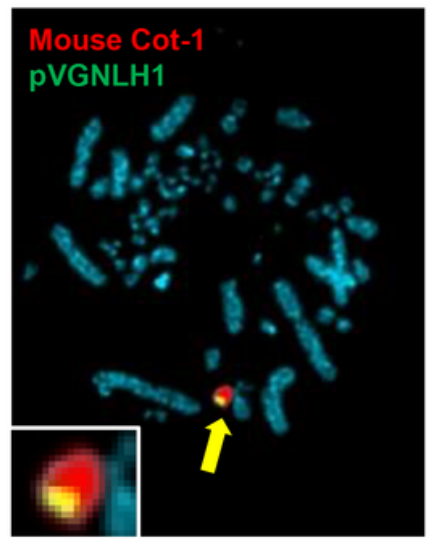

\section{Figure 2}

Strategy for the targeted truncation and construction of 10MAC1 vector (A) Fluorescence in situ hybridization (FISH) analysis with a digoxigenin-labeled mouse Cot-1 DNA (red) identified the intact 
mouse chromosome 10 in the DT40 cells. The arrow indicates an intact mouse chromosome 10 and the inset shows an enlarged image. The chromosomal DNA was counterstained with DAPI, and DAPI was used for the same purpose in the following FISH experiments. (B) Strategy for the targeted truncation of the distal region of mouse chromosome 10 by the targeting vector, pBS-TEL/puro_10MAC. The telomereseeding vector was electroporated into DT40 cells containing mChr.10-NeoR to yield puromycin-resistant transfectants. Arrowheads indicate genomic PCR primers. (C) A two-color FISH probe comprising the mouse Cot-1 DNA (red) that hybridizes with the 10MAC and puromycin-resistant gene (green) localized to the distal end of truncated 10MAC. The arrow indicates the MAC fragment, and the inset shows an enlarged image. (D) Strategy for the targeted integration of the EGFP gene, NeoR and 3'HPRT-loxP into the 10MAC to construct 10MAC1 by the targeting vector, p10MAC1. Arrowheads indicate genomic PCR primers. (E) Electroporation of the pMAC1 plasmid yielded GFP-expressing, G418-resistant transfectants from the DT40 (10MAC1) cells. Phase-contrast (top panel) and fluorescence (bottom panel) micrographs are shown. Scale bar: $100 \mu \mathrm{m}$. FISH analysis of the 10MAC1 vector in the DT40 (10MAC1) cells using the digoxigenin-labeled mouse Cot-1 DNA (red) and the biotin-labeled pVGNLH1 (green). The arrow indicates $10 M A C 1$, and the inset shows an enlarged image of 10MAC1.

A

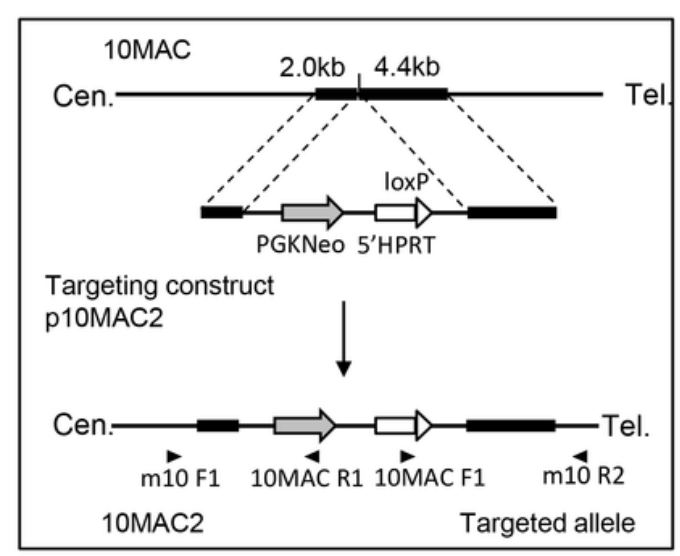

B

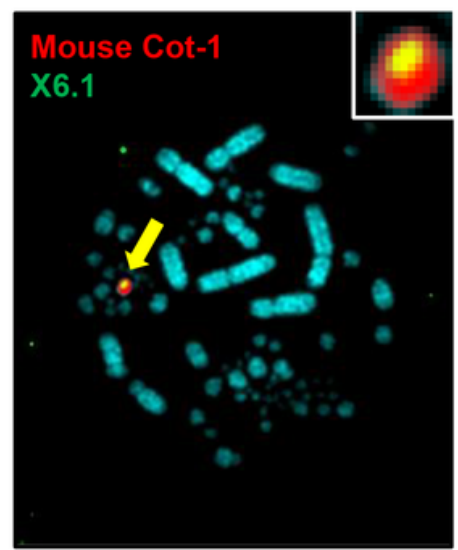

C

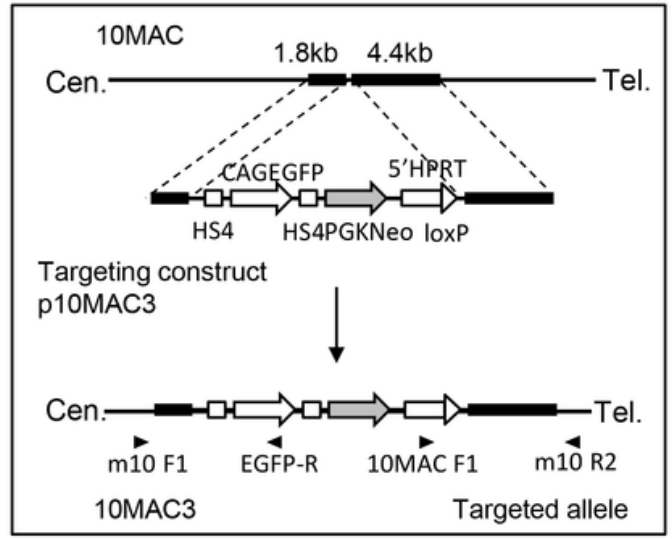

D

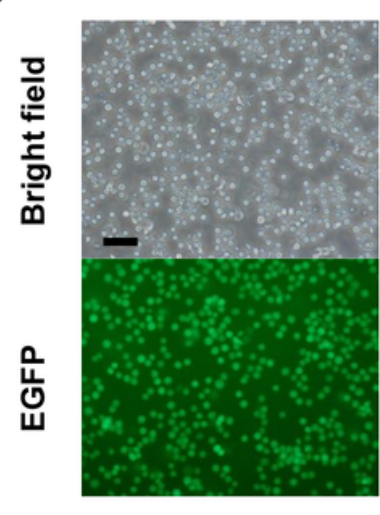

$\mathbf{E}$

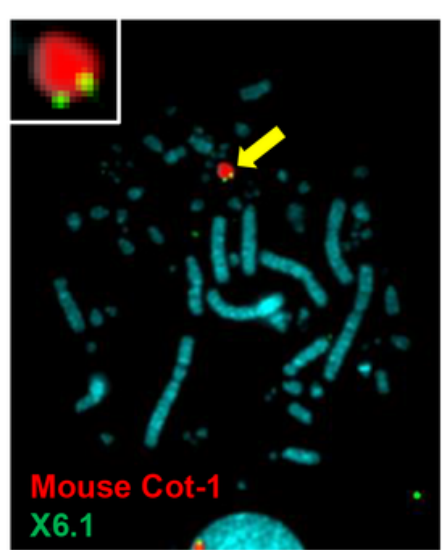

Figure 3 
Strategy for the construction of 10MAC2 and 10MAC3 vectors (A) Strategy for the targeted integration of NeoR and loxP-5'HPRT into the 10MAC to generate 10 MAC2 vector by the targeting vector, p10MAC2. Arrowheads indicate genomic PCR primers. (B) FISH analysis of the 10MAC2 vector in the DT40 (10MAC2) cells using the digoxigenin-labeled mouse Cot-1 DNA (red) and the biotin-labeled loxP-5'HPRT gene (green). The arrow indicates the $10 \mathrm{MAC} 2$ vector, and the inset shows an enlarged image of the 10MAC2 vector. (C) Strategy for the targeted integration of EGFP, NeoR and loxP-5'HPRT into the 10MAC to generate $10 \mathrm{MAC} 3$ vector by the targeting vector p10MAC3. Arrowheads indicate genomic PCR primers. (D) Electroporation of the pMAC3 plasmid yielded GFP-expressing, G418-resistant transfectants from the DT40 (10MAC3) cells. Phase-contrast (top panel) and fluorescence (bottom panel) micrographs are shown. Scale bar: $100 \mu \mathrm{m}$. (E) FISH analysis of the 10MAC3 vector in the DT40 (10MAC3) cells using the digoxigenin-labeled mouse Cot-1 DNA (red) and the biotin-labeled loxP-5'HPRT gene (green). The arrow indicates the 10MAC3 vector, and the inset shows an enlarged image of the 10MAC3 vector.

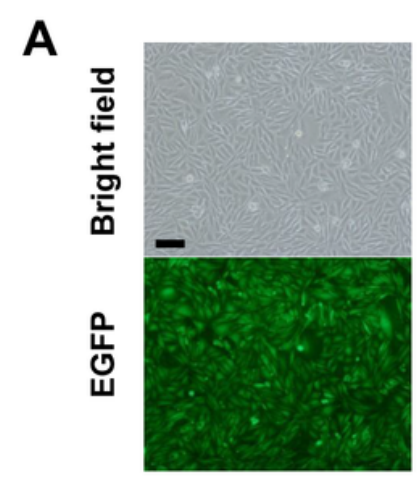

\section{B}
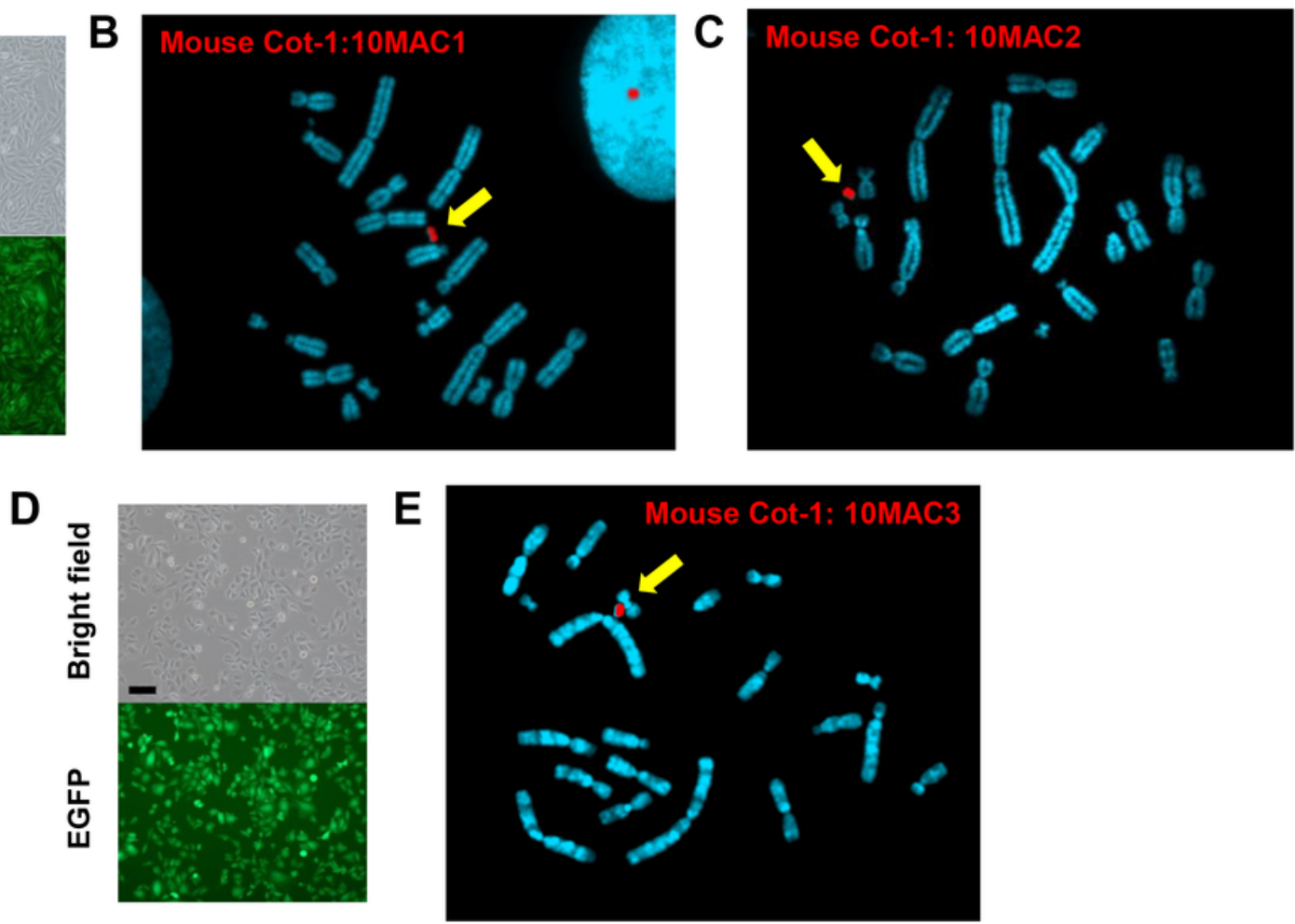

\section{Figure 4}

Transfer of 10MAC vectors to $\mathrm{CHO}$ (hprt-/-) (A) and (D) Phase-contrast (top panel) and fluorescence (bottom panel) micrographs of $\mathrm{CHO}$ (10MAC1) and $\mathrm{CHO}$ (10MAC3) are shown. Scale bar: $100 \mu \mathrm{m}$. (B), (C) 
and (E) FISH analyses of each 10MAC vector in the CHO (10MAC1), CHO (10MAC2) and CHO (10MAC3) by digoxigenin-labeled mouse Cot-1 DNA. Arrows indicate each 10MAC vector.

A

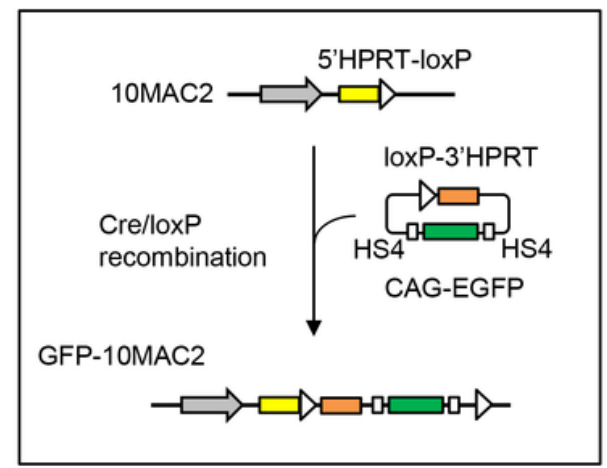

D

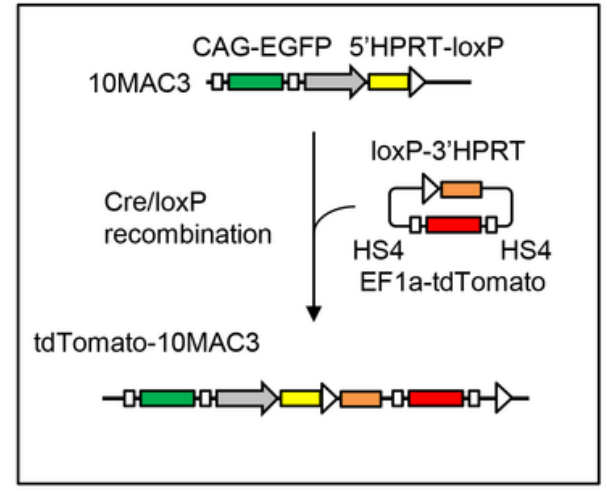

B

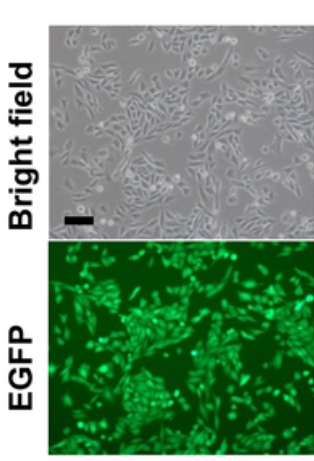

E

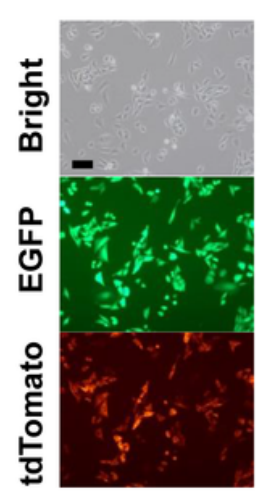

C

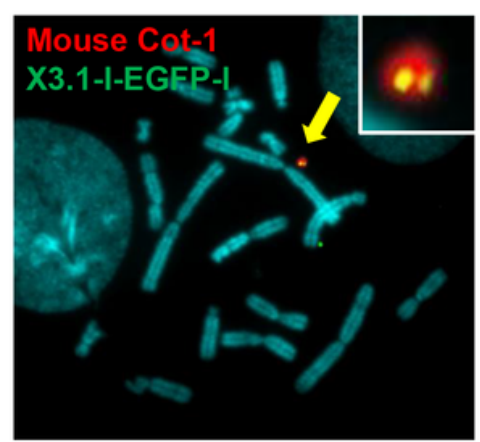

$\mathbf{F}$

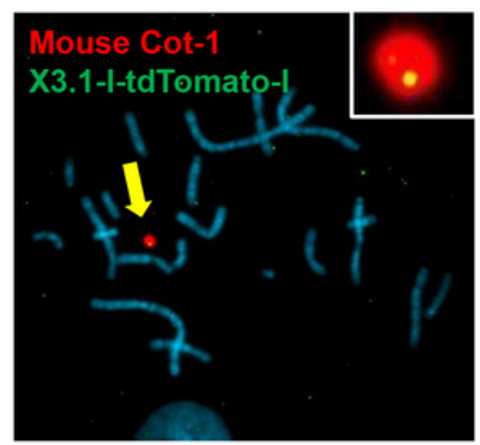

\section{Figure 5}

Cre/loxP-mediated gene loading to the 10MAC2 and 10MAC3 (A) and (D) Strategy for the site-specific insertion of the EGFP and tdTomato genes into the 10MAC2 and 10MAC3 vectors, respectively, using the Cre/loxP-mediated system in $\mathrm{CHO}$ cells. (B) Transfection yielded GFP-expressing HAT-resistant transfectants from the $\mathrm{CHO}$ (10MAC2) cells. Phase-contrast (top panel) and fluorescence (bottom panel) micrographs are shown. Scale bar: $100 \mu \mathrm{m}$. (C) FISH analysis of the GFP-10MAC2 in the CHO (GFP10MAC2) cells using the digoxigenin-labeled mouse Cot-1 DNA (red) and the biotin-labeled EGFP gene (green). The arrow indicates the GFP-10MAC2 vector, and the inset shows an enlarged image of the GFP$10 \mathrm{MAC} 2$ vector. (E) Transfection yielded tdTomato-expressing HAT-resistant transfectants from the $\mathrm{CHO}$ (10MAC3) cells. Phase-contrast (top panel) EGFP (middle panel) and tdTomato (bottom panel) micrographs are shown. Scale bar: $100 \mu \mathrm{m}$. (F) FISH analysis of the tdTomato-10MAC3 in the CHO (tdTomato-10MAC3) cells using the digoxigenin-labeled mouse Cot-1 DNA (red) and the biotin-labeled 
tdTomato gene (green). The arrow indicates the tdTomato-10MAC3 vector, and the inset shows an enlarged image of the tdTomato-10MAC3 vector.

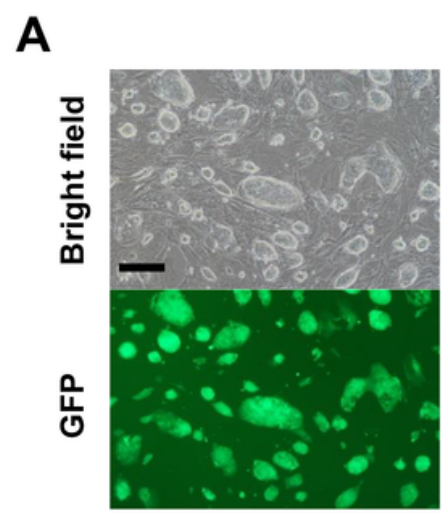

$\mathbf{E}$

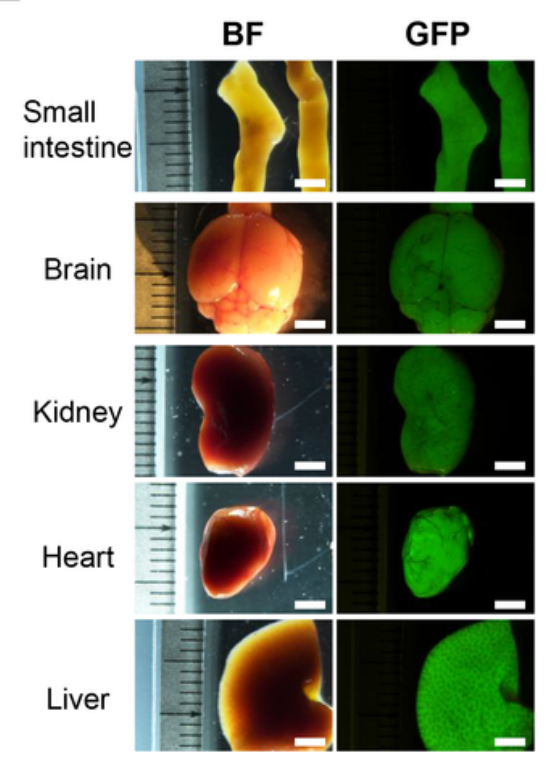

B

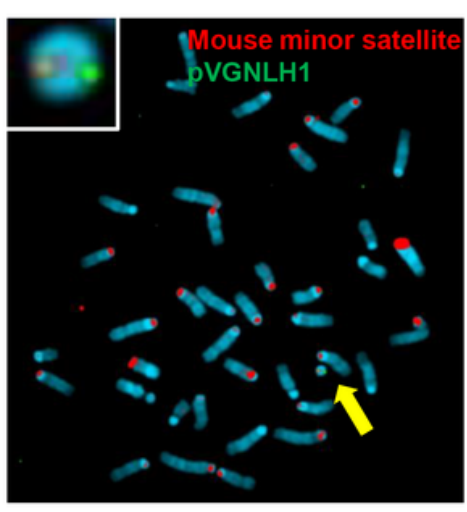

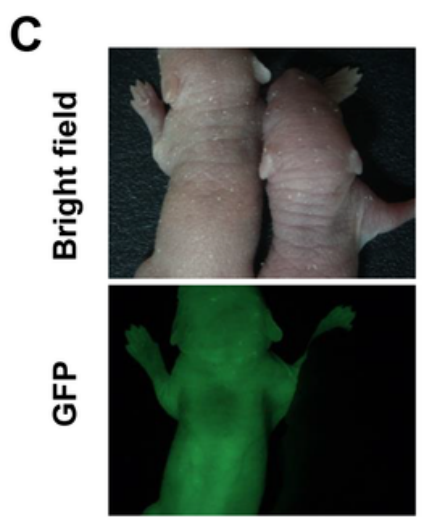

F
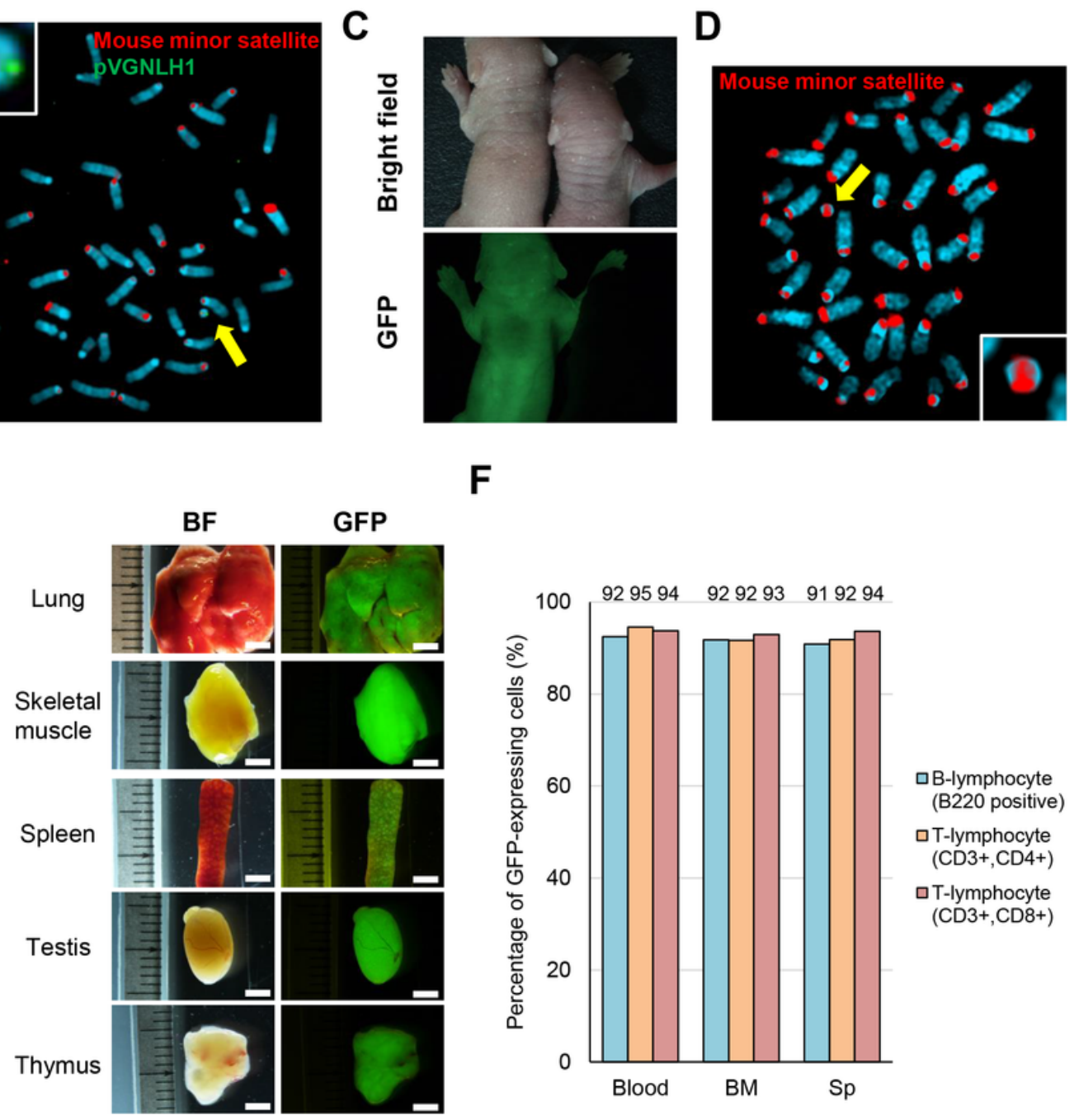

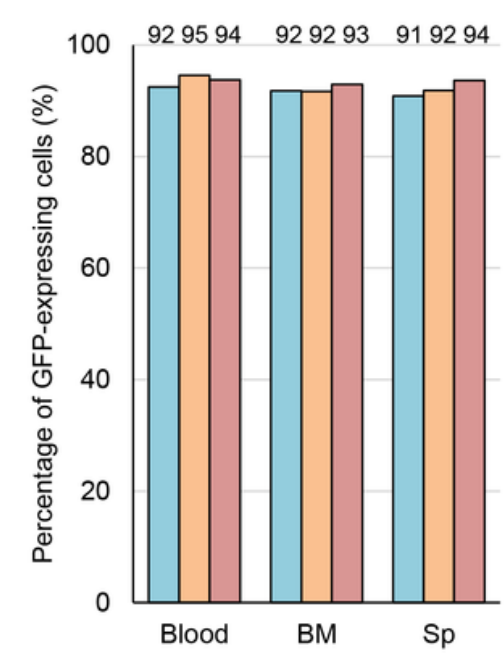

口B-lymphocyte (B220 positive) 口T-lymphocyte (CD3+,CD4+) $\square$ T-lymphocyte (CD3+,CD8+)

\section{Figure 6}

Generation of Tc mice carrying 10MAC1 and its stability in the tissues (A) Phase-contrast (top panel) and fluorescence (bottom panel) micrographs of mouse ES hybrids carrying 10MAC1 are shown. Scale Bar: $100 \mu \mathrm{m}$. (B) FISH analysis of the mouse ES hybrids carrying 10MAC1 using the digoxigenin-labeled mouse minor satellite DNA (red) and the biotin-labeled pVGNLH1 (green). Inset shows an enlarged image of the 10MAC1. (C) Phase-contrast (top panel) fluorescence (bottom panel) micrographs of wild-type (right) and Tc mice (left) are shown. (D) Representative FISH image of metaphase chromosome spreads of lymphocytes in blood from Tc mice using the digoxigenin-labeled mouse minor satellite DNA (red). Inset shows an enlarged image of the 10MAC1. (E) GFP images of different tissues from Tc mice carrying the 10MAC1. GFP expression indicates the presence of 10MAC1. Exposure times for each GFP image of small intestine, brain, kidney, heart, liver, lung, skeletal muscle, spleen, testis, and thymus were 100, 500, 200, 100, 400, 700, 100, 400, 200 and 200 ms, respectively. Scale bar: 3 mm. (F) Percentage of GFPexpressing lymphocyte subsets from blood, bone marrow and spleen. 


\section{Supplementary Files}

This is a list of supplementary files associated with this preprint. Click to download.

- Abeetal.Supplementarylnformation.pdf 